\title{
La competencia comunicativa con fines profesionales desarrollada mediante tareas
}

\begin{abstract}
Máster Jane da Silva Amorim', Instituto Federal de Roraima, Brasil y Máster Leila Márcia Ghedin², Instituto Federal de Roraima, Brasil
\end{abstract}

$\begin{array}{ll}\text { Recibido: } & 19 \text { de abril, } 2017 \\ \text { Aceptado: } & 3 \text { de octubre, } 2017\end{array}$

\section{Resumen}

En este artículo se describen y analizan tareas realizadas en un contexto de instrucción formal de brasileños aprendices de español como Lengua Extranjera (LE) con propósitos profesionales apoyadas tanto en la teoría como en la práctica. En cuanto a la teoría de modo general, el estudio se ha basado en los presupuestos teóricos de la Lingüística Aplicada (LA) como ciencia interdisciplinar en la que se puede encuadrar también, aportes de la Investigación-Acción, teorías cognitivas de aprendizajes, métodos y enfoques comunicativos. Con respecto a la práctica, se describen y analizan las tareas que fueron elaboradas y aplicadas a un grupo de estudiantes brasileños de español LE con propósitos profesionales: Letras Español, con edades entre 18 y 40 años del Módulo V - año académico 2017.2 del Instituto Federal de Roraima (IFRR),en que se comprueba las teorías utilizadas. Se trata de un estudio descriptivo de cuño exploratorio de naturaleza cualitativa que combina criterios observacionales y narrativos. Como resultado, se ha comprobado que unidades didácticas diseñadas mediante tareas con aportes de la investigaciónacción contribuyen a un desarrollo eficaz de las destrezas básicas de aprendizaje del español con propósitos profesionales. Del mismo modo, los resultados permitieron cubrir carencias de datos espontáneos y experimentales con relación al problema de desarrollo en el aprendizaje de E/LE con fines específicos.

\section{Abstract}

\section{Communicative competency with professional purpose developed through tasks}

This article describes and analyzes tasks carried out by Brazilian students of Spanish for Professional Purposes as a foreign language $(\mathrm{FL})$ in a formal instructional context supported in theory and practice. This study is based on the assumptions of applied linguistics (AL) as an interdisciplinary science, action research contributions, and cognitive theories of learning, methods and communicative approaches. It describes and analyzes the tasks developed and carried out by a group of Brazilian students of Spanish (FL) for Professional Purpose ages 18 to 40 of the V module of 2017 academic year of the Instituto Federal de Roraima (IFRR) proving the theories applied. It is a descriptive, exploratory, and qualitative study that combines observational and narrative criteria. The results show that the didactic units designed through tasks with research insights contribute to the effective development of the basic Spanish for Professional Purposes learning skills. In the same way, results allowed to cope with the lack of spontaneous and experimental data related to the development problem in E/LE learning with specific purposes

1 Jane da Silva es máster en la Enseñanza de Español como Lengua Extranjera, graduada en la Universidad Antonio de Nebrija, España. Actualmente se encuentra pensionada. Contacto: enajamorim@gmail.com.

2 Leila Márcia Ghedin es profesora del Instituto Federal de Roraima, Brasil. Doctoranda en Educación de Ciencia y Matemática en la Red de Educación en Ciencia y Matemática (REAMEC) y máster en Educación de Ciencias, graduada de la Universidad Estatal de Amazonas, Brasil. Contacto: leilaghedin@ifrr.edu.br.
Jane da Silva Amorim y Leila Márcia Ghedin. La competencia comunicativa con fines profesionales desarrollada mediante tareas. Revista Comunicación. Año 38, volumen 26, número 2, julio-diciembre, 2017. Instituto Tecnológico de Costa Rica. ISSN: 0379-3974 / e-ISSN1659-3820.

\section{PALABRAS CLAVE:}

Enseñanza de idiomas, lenguas extranjeras, método de aprendizaje, aprendizaje mediante la práctica, trabajos prácticos.

\section{KEY WORDS:}

Teaching Idiom, Foreign Language, Learning Method, Learning through practice, practical work. 


\section{INTRODUCCIÓN}

\section{Origen de la investigación}

Esta investigación surge a partir de la experiencia de las autoras, como profesoras de lengua española como lengua extranjera E/LE - en cursos con fines específicos (técnicos o superiores: Letras - Español, Gestión Hospitalaria, Secretariado, Turismo entre otros) unida a la inquietud de desarrollarse en la construcción del conocimiento, de manera más efectiva, de modo que se propiciara un aprendizaje reflexivo y autónomo de la lengua española como LE.

Basándose en ese indicio, se buscaron estudios sobre el proceso de enseñanza-aprendizaje del español como lengua extranjera (E / LE), teniendo como base la investigación - acción y las tareas como estrategias didácticas en curso con propósitos profesionales. Gracias a esto se advirtió que existen pocos estudios que tienen como base ese conjunto, ya sea en lengua española o portuguesa. Entre los que se han podido consultar destacan los estudios de Stenhouse (1998), McKernan (1999), Nunan (2004 y 1998), Estaire (1999), Baralo (2000), Baralo y Estaire (2010), Estaire y Zanón (1990) y Sansoles (2010).

Esta escasez de investigación sobre el tema dio lugar a una búsqueda más exhaustiva y profunda en el área de la investigación acción y tareas, así que con base en esa temática fue necesario adoptar algunos procedimientos. El primero fue revisar la bibliografía sobre la investigación acción como base de la enseñanza y las tareas como estrategias didácticas en el proceso de enseñanza aprendizaje de E/LE en cursos con propósitos profesionales, lo cual solo es posible haciendo una investigación de otras ciencias que comparten el mismo objeto de estudio, entre ellas la psicolingüística y la sociolingüística cognitiva, entre otras.

El objetivo fundamental de este trabajo fue describir y analizar tareas realizadas en un contexto de instrucción formal de brasileños aprendices de Español LE con propósitos profesionales, para lo cual se utilizó tanto la teoría como la práctica. En cuanto a la teoría de modo general el estudio se basó en los presupuestos teóricos de la Lingüística Aplicada (LA) como ciencia interdisciplinar en la que se pueden encuadrar también aportes de la Sociolingüística Cognitiva (SG), Psicolingüística e Investigación-Ac- ción, además de elementos del español con fines profesionales. Con respecto a la práctica, se elaboró una unidad didáctica mediante el enfoque por tarea que fue aplicada a un grupo de estudiantes brasileños de español LE con propósitos profesionales de la carrera Letras - Español, V módulo, año académico 2017/2 del Instituto Federal de Roraima, Brasil (IFRR), en que se comprueba las teorías utilizadas.

Para lograr el objetivo se usó una metodología basada en técnicas de observación y registro en el desarrollo de las tareas en el aula. En concreto, se seleccionó un grupo de 17 brasileños estudiantes de E / LE alumnos de la carrera Letras Español, con edades entre 18 y 40 años. Para este estudio en particular, fueron cuidadosamente controladas las características lingüísticas y sociales de los sujetos de la investigación. Se aplicó una unidad didáctica elaborada con fundamentos del enfoque por tarea en el aprendizaje de lengua extranjera. Esta unidad sirvió de base para la descripción y análisis de las micro-tareas y tarea final, objeto de estudio.

Para esta investigación se partió de la siguiente premisa: Si la competencia comunicativa con fines profesionales llevada a cabo mediante tareas es efectiva para el aprendizaje de E / LE, entonces las personas involucradas desarrollarán eficazmente las destrezas básicas del aprendizaje del español en la unidad didáctica aplicada.

Dando seguimiento al proyecto, se planteó la siguiente pregunta de investigación: ¿Las tareas como estrategias didácticas contribuyen a un aprendizaje efectivo del español LE en cursos con propósitos profesionales?

Con las informaciones recopiladas se pudo explicar, describir y analizar la unidad didáctica basada en el enfoque por tareas en el aprendizaje de E/LE con fines profesionales. Se cree que la investigación, de modo incipiente, aportará conocimientos sobre las bases lingüísticas, sociolingüísticas cognitivas, investigación acción y estrategias didácticas mediante tareas para la sistematización de unidades didácticas a ser desarrolladas en cursos con fines profesionales y en un contexto formal de aprendizaje. Igualmente, la investigación contribuirá a la ampliación de datos espontáneos y experimentales con relación al problema de desarrollo en el aprendizaje de E/LE con fines específicos. 


\section{MARCO TEÓRICO}

\section{La construcción del conocimiento}

Para la teoría del aprendizaje, la psicología cognitiva presenta varias alternativas que se aproximan a las estructuras y funciones de la vida humana, esto por medio de representaciones mentales, manipulación y transformación de información. En este sentido, se hace inevitable abordar la definición de construcción y producción del conocimiento para aclarar el camino que se quiere recorrer. Para Pozo (1987), citado en Fabregat y Reig (1998, p.74), la "diferencia central de esas estructuras y funciones cognitivas, reside en la unidad básica de análisis de la que parten, mientras que unas parten de unidades mínimas, otros parten de unidades más amplias". De esta forma, se puede decir que el constructivismo cognitivo configura las representaciones de la realidad y se extiende hacia las posibilidades de transformaciones de las experiencias empíricas en la producción de conocimiento.

No obstante, en primer lugar es importante preguntarse ¿qué es conocimiento y cómo sucede en el aula? El conocimiento es todo lo que el ser humano aprende, transforma y usa según su necesidad. Al tratar este concepto en el aula, es posible afirmar que los estudiantes poseen una variedad de conocimientos que traen de su realidad. Así, el contexto en que viven sus experiencias puede propiciar una cantidad mayor o menor de variaciones de los aspectos de la realidad (Miras, 2003, p. 63).

La misión de los profesores es traer a la luz estas representaciones e involucrarlas con los contenidos aplicados en el aula para que los estudiantes construyan sus conocimientos y posteriormente produzcan los conocimientos provenientes de esta actividad. Se entiende como construcción del conocimiento el proceso que ocurre -en este caso- en el aula y propicia la aprehensión de los conocimientos, esto es, todo lo que el estudiante logre captar y guardar para sí podrá transformarlo y utilizarlo en otras situaciones semejantes. En otras palabras, la producción del conocimiento es la manifestación publicación de estos conocimientos provenientes de experiencias que el estudiante vivió, por medio de artículos, presentaciones en eventos científicos, libros y actividades desarrolladas en el aula, entre otros.

\section{Competencia comunicativa y procesos de aprendizaje}

Muchos son los estudios que tratan de explicar el proceso de enseñanza y aprendizaje por el cual pasan los estudiantes de una lengua para lograr aprenderla, siendo esta una lengua segunda (L2) o Lengua Extranjera (LE).

Para este estudio se usaron las definiciones de Baralo (1999) cuando se refiere a L2 y LE. Suele llamarse L2 a la lengua que se aprende en comunidades que disponen de dos sistemas lingüísticos en contacto LE es la lengua que se aprende en un contexto institucional. Como cualquier tipo de aprendizaje, la adquisición de una L2 o LE es una acumulación de conocimientos, sean estos fonéticos, sintácticos, semánticos y pragmáticos, los cuales requieren mucha práctica y habilidades.

Para comprender el proceso de aprendizaje de una lengua extranjera es necesario observar conceptos como competencia comunicativa, que es "un conocimiento intuitivo, no consciente de una extraordinaria complejidad y abstracción, que nos permite comunicarnos con eficacia en una lengua determinada" (Baralo, 1998, p. 15). Teóricos como Hymes (1972) aportan el concepto de competencia comunicativa, ampliando la definición de competencia lingüística propuesta por Chomsky. Según Hymes, la competencia comunicativa es el conjunto de habilidades y conocimientos que posibilitan a una comunidad lingüística participar eficaz y adecuadamente en una interacción social de una sociedad determinada; es decir, implica respetar un conjunto de reglas, que incluye tanto las de la gramática y los otros niveles de la descripción lingüística (léxico, fonética, semántica) como las reglas de uso de la lengua relacionadas con el contexto socio-histórico y cultural en el que tiene lugar la comunicación. Desde una visión etnográfica de la comunicación, especifica que la competencia comunicativa se relaciona con saber "cuándo hablar, cuándo no, de qué, con quién, cuándo, dónde, en qué forma"

\section{Métodos y enfoques comunicativos}

En lo referente a métodos y enfoques comunicativos eficaces se destaca el enfoque por tarea, que es:

[...] la propuesta de un programa de aprendizaje de lengua cuyas unidades consisten 
en actividades de uso de la lengua, y no en estructuras sintácticas (como hacían los métodos audiolinguales) o en nociones y funciones (como hacían los programas nociofuncionales). Su objetivo es fomentar el aprendizaje mediante el uso real de la lengua en el aula y no solo mediante manipulación de unidades de sus diversos niveles de descripción. (Centro Virtual Cervantes (CVC, 1997-2017).

Long (citado en Estaire y Zanón, 1990) expone que una tarea

[es] cualquier actividad realizada, por uno mismo o para los demás, libremente o con algún interés. [...] rellenar un impreso, comprar unos zapatos, hacer una reserva de avión [...] en otras palabras, por "tareas" entendemos todo aquello que solemos hacer en nuestra vida cotidiana, en el trabajo, para divertirnos y entre una y otra cosa (p. 63).

Baralo (2000), por su parte, afirma que las tareas son actividades diseñadas para que el aprendiente use la lengua comunicativamente con algún propósito concreto, o de forma reflexiva, para resolver algún problema, conseguir alguna información y transmitirla, tomar decisiones según diferentes argumentos (pp. 18-19).

Desde una perspectiva pedagógica, Baralo y Estaire (2010) opinan que

Este modelo didáctico se basa en una concepción cognitiva/constructivista del aprendizaje de lenguas extranjeras, que subraya el papel de la actividad mental constructiva del alumno, inmerso en un proceso continuo de construcción, reestructuración y apropiación de conocimientos, lo que le convierte en agente activo de su propio aprendizaje.

A lo largo de sus años de docencia, quienes escriben han llegado a la conclusión de que una de las bases del aprendizaje de cada alumno es hacerlo de forma que pueda "aprender haciendo". Para lograrlo, una de las estrategias didácticas más efectivas está en desarrollar microtareas y culmina con una tarea final, sin olvidar en ningún momento como profesores los objetivos pedagógicos específicos que todo diseño de estrategias didácticas conlleva ni la pro- puesta interdisciplinar que ofrece el soporte para el buen desarrollo de cualquier componente curricular del programa o curso.

Para conseguir el reto de "aprender haciendo" siempre debe buscarse bases que apoyen la labor docente, entre ellas, la investigación-acción. Al respecto, Elliott (citado en McKernan, 1999) afirma que la investigación-acción es "el estudio de una situación social con miras a mejorar la calidad de la acción dentro de ella" (p. 24). Por su parte, Stenhouse (también, citado en McKernan, 1999) define la investigación como el "estudio sistemático y sostenido, planificado y autocrítico que está sujeto a la crítica pública y a pruebas empíricas, cuando estas son apropiadas" (p. 25).

En una definición mínima de la investigación-acción, McKernan (1999) señala:

La investigación acción es el proceso de reflexión por el cual en un área problema determinada, donde se desea mejorar la práctica o la comprensión personal, el profesional en ejercicio lleva a cabo un estudio, en primer lugar, para definir con claridad el problema; en segundo lugar, para especificar un plan de acción, que incluye el examen de hipótesis por la aplicación de la acción al problema. Luego se emprende una evaluación para comprobar y establecer la efectividad de la acción tomada. Por último, los participantes reflexionan, explican los progresos y comunican estos resultados a la comunidad de investigadores de la acción. La investigación-acción es un estudio científico autorreflexivo de los profesionales para mejorar la práctica (p. 25).

Específicamente, la unidad didáctica fue desarrollada en áreas especializadas: Letras Español, salud, turismo, hospitalidad y secretariado, entre otros. Para desarrollar actividades en estas áreas era necesario conocer las necesidades reales de los alumnos para que pudieran interactuar de manera fluida y así lograr el uso de la lengua en su sector específico de actuación. Asimismo, esta propuesta está basada en estudios de la lengua española con fines específicos y por tanto posee características distintas del español en "general". La denominación "Español con fines específicos" empleada a comienzos de los años 
noventa para referirse a los procesos de enseñanza aprendizaje que tienen como objetivo la adquisición de la lengua que es propia de la comunicación especializada (Gómez Enterría, 2006).

\section{METODOLOGÍA}

\section{Caracterización del espacio en que se ha realizado la investigación}

El Estado de Roraima se encuentra en el extremo norte de Brasil e integra la Amazonía Occidental. Limita al norte con la República Bolivariana de Venezuela, al sur con los Estados de Amazonas y Pará, y al este con la República Cooperativista de Guyana. Su población está conformada por personas provenientes de todas las regiones del país, además de su fuerte influencia étnica y cultural indígena. Se compone de quince municipios entre ellos, la capital Boa Vista, la única en Brasil situada encima de la línea del Ecuador, una ciudad moderna y atractiva, planificada y trazada en forma de abanico, a través de un proyecto arquitectónico inspirado en la antigua París. La población residente en la capital está estimada en 314.900 personas, según datos del Instituto Brasileño de Geografía y Estadística (IBGE, 2014).

La ciudad de Boa Vista nació en el siglo XIX cuando las haciendas comenzaron a erigirse a la orilla del río Blanco, en el entonces llamado pueblo de la Freguesia de Nuestra Señora del Carmen y hoy es sin duda una de las más bellas de la región norte del país. Además es la única capital brasileña ubicada totalmente en el hemisferio norte del planeta, un lugar tranquilo, con un pueblo alegre y acogedor. Su ubicación, a las márgenes del río Blanco y su clima tropical de altas temperaturas forman una combinación perfecta para el turismo local. Esta ciudad vive actualmente un crecimiento notable y se encuentra en plena expansión con lo que atraen emprendimientos de conceptos innovadores. El sector de servicios es uno de los más influyentes de Boa Vista según datos de la Secretaría de Estado de Planificación y Desarrollo (SEPLAN, 2014).

La investigación se realizó en el Instituto Federal de Educación, Ciencia y Tecnología de Roraima (IFRR). El IFRR es una institución de enseñanza básica, técnica, superior (licenciatura y tecnológica) y de postgrado. En la enseñanza técnica se desarrollan cursos en el área de industria, gestión y salud. En la graduación se realizan cursos de licenciaturas y tecnologías. La institución nació a partir de la Escuela Técnica Estatal que se federalizó en 1993, en aquel momento se ofrecía enseñanza fundamental, secundaria y técnica. En el 2002 se transformó en el Centro Federal de Educación Tecnológica de Roraima (CEFET-RR) cuando se ofreció el primer curso superior, siendo este, el Curso de Tecnología en Gestión de Turismo. En el 2008 por medio de la Ley 11.892 el Instituto Federal de Educación, Ciencia y Tecnología de Roraima con el objetivo de impartir educación profesional técnica de nivel medio y cursos de formación inicial y continuada a trabajadores realizó investigación para desarrollar actividades de extensión, además de ofrecer cursos de postgrado lato sensu de perfeccionamiento y especialización, así como cursos de postgrado stricto sensu de maestría y doctorado. Ofrece cursos en diversas modalidades y niveles de enseñanza. Como instituto debe promover la enseñanza, la investigación y la extensión (IFRR, 2017).

Con respecto a la investigación Kut Levin (citado en McKernan, 1999, p.23) afirma que "la investigación que no produce más que libros no será suficiente". Sea cual sea el campo o el nivel elegido, el investigador necesita un método de recopilación y análisis de los datos, ya sea de tipo cuantitativo o cualitativo. Entre los métodos cualitativos más empleados se encuentran la observación de campo, los grupos de enfoque, el análisis del discurso y el estudio de casos. De los métodos cuantitativos destacan el análisis de contenido, las encuestas, la investigación longitudinal y la investigación experimental (Wimmer y Dominick, 2001).

La metodología propuesta podría integrarse en los estudios Ilamados investigación de campo observacionales y narrativos. Se ha elegido el estilo observacional no participante en el cual el investigador es "poco visible y no se compromete en los roles y el trabajo del grupo como miembro de él" (McKernan, 1999, p. 81).

El análisis se realizó tras la aplicación de una unidad didáctica con aportes del enfoque por tarea a un grupo de 17 alumnos, con edades entre 18 y 40 años, aprendices de español como LE en la carrera Letras Español y sus literaturas. Los participantes eran del módulo $\vee$ del año académico de 2017-2 del IFRR. La unidad se desarrolló en 6 horas con clases de 45 minutos cada una. 
Así pues, se diseñaron algunas tareas centradas en el significado para que los alumnos brasileños de español E/LE con propósitos profesionales lograron desarrollarlas y concretarlas con una tarea final. Los objetivos específicos se enfocaron en la producción de las actividades adecuadas para la enseñanza de español a un luso hablante con fines profesionales, además de experimentar el dominio cognitivo por parte de alumnos y profesores con respecto a contenidos vistos en clase.

La unidad didáctica elaborada está estructurada bajo la combinación del español LE con fines profesionales para el área de Letras Español y en este sentido fue pensada para que su producto fuera considerado útil por parte del alumno, relacionado su práctica profesional, que es la docencia.

Como se mencionó en el apartado anterior, el estudio se enmarcó dentro de las bases del enfoque por tareas como estrategias didácticas en el proceso de enseñanza y aprendizaje del español como LE en contexto formal de aprendizaje. Se incluyeron también los fundamentos de la investigación acción presentes en las metodologías cualitativas.

La elaboración de la unidad didáctica mediante tareas se inspiró en los pasos producidos por Estaire y Zanón (1990):

Figura 1: Pasos que deben seguir los docentes, en solitario o en conjunción con los alumnos

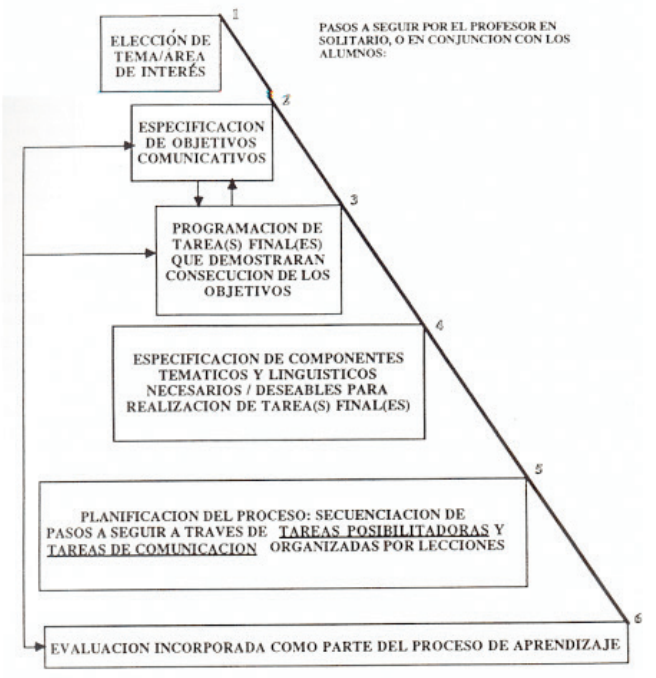

Fuente: Marco para la elaboración de unidad didáctica a través de tareas elaborada por Estaire, S. y Zanón, J. (1990). El diseño de unidades didácticas mediante tareas: principios y desarrollo. Comunicación, Lenguaje y Educación, 7.

\section{Unidad didáctica elaborada por las autoras}

Tarea final: Producción de una Carta Enigmática

Destrezas: Expresión oral, expresión escrita, comprensión lectora y compresión auditiva

Objetivo comunicativo: Interacción oral y de escritura

Objetivo gramatical: Revisar verbos, oraciones comparativas, definiciones...

Nivel: B2 y C1 (intermedio superior inicial)

Duración: Entre 4 y 6 horas con clases de 45 minutos cada una

Material: Copias del texto: canción No importa la distancia (Ricky Martin)

Desarrollo: Todos los participantes ejecutaron las microtareas siguientes que fueron elaboradas por las autoras.

\section{Microtarea 1}

1.1. Antes de escuchar la canción, intenta completar los espacios en blanco con las palabras correspondientes a las siguientes definiciones:

1. No se emplea sino antepuesto a nombres masculinos. Es antónimo de NINGUNO.

2. Pronombre indefinido que designa persona $\mathrm{o}$ personas existentes, sin indicación de género ni de número. Es antónimo de NADIE.

3. Verbo transitivo que en una de sus definiciones significa "conseguir o alcanzar lo que se intenta o desea".

4. Como verbo intransitivo significa "Salir victorio$\mathrm{SO}^{\prime \prime}$.

5. Exceso de estimación propia que a veces es disimulable por nacer de causas nobles y virtuosas.

6. Artículo indeterminado en género masculino y número singular.

7. En una semana hay siete.

8. Artículo indeterminado en género femenino y número singular.

9. Pronombre indeterminado. No se emplea sino antepuesto al nombre.

10. Conjunción concesiva y adversativa que en lengua portuguesa significa: A PESAR DE / AINDA QUE. 
11. El que lleva a cabo una acción heroica.

12. Verbo intransitivo que significa "alcanzar el fin o término de un desplazamiento".

\section{NO IMPORTA LA DISTANCIA}

\section{(Ricky Martin) Fuente: Google}

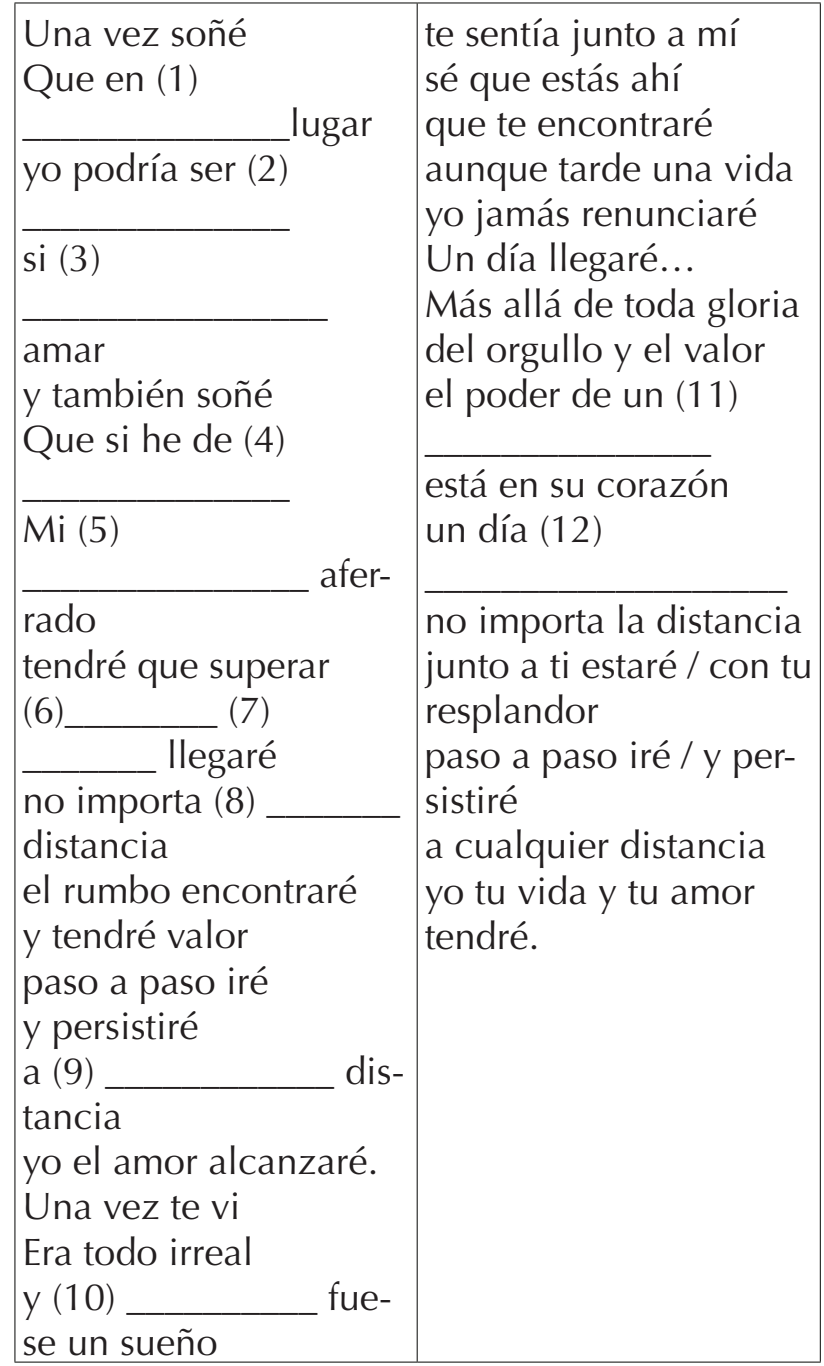

Microtarea 2

2.1 Ahora escucha la canción y comprueba si lo que has escrito es correcto. Luego saca de la canción 10 palabras o expresiones que en tu opinión son importantes para la compresión del mensaje que transmite.

\section{Microtarea 3}

3.1. Elabora un párrafo con las palabras o expresiones que sacaste del texto.

\section{Microtarea 4}

4.1 En esta canción:

1. "El rumbo encontraré" significa:

- Cualquier agujero que se hace o se produce en el casco de la nave.

- Camino y senda que uno se propone a seguir para llegar a un lugar o fin determinado.

- Pompa, ostentación y aparato costoso.

2. "Aunque tarde una vida" significa:

- Últimas horas del día.

- Tiempo que hay desde mediodía hasta anochecer.

- Tiempo futuro relativamente lejano.

4.2 Haz frases siguiendo el modelo comparativo: más...que.

La humildad - importante - orgullo - La humildad en más importante que el orgullo

$$
\begin{aligned}
& \text { El - corazón - héroe - fuerte - distancia } \\
& \text { La persistencia - importante - renuncia }
\end{aligned}
$$

Tarea Final: Producir un texto ilustrativo con dibujos imaginando que la canción es una carta que has recibido de un (a) gran amigo (a). Presentar a los demás participantes.

Después de ejecutar las tareas secundarias los alumnos elaboraron y presentaron la tarea final.

\section{Ejemplo de la tarea final, elaborada por una alumna del módulo $\mathrm{V}$-Letras y Literatura Hispánica- IFRR, 2017, 2}

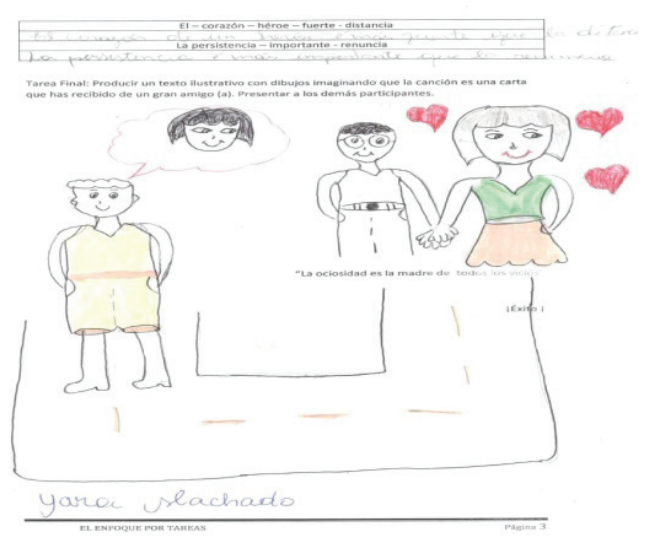




\section{Ejemplo de la tarea final, elaborada por una alumna del módulo $\mathrm{V}$-Letras y Literatura Hispánica- IFRR, 2017, 2}

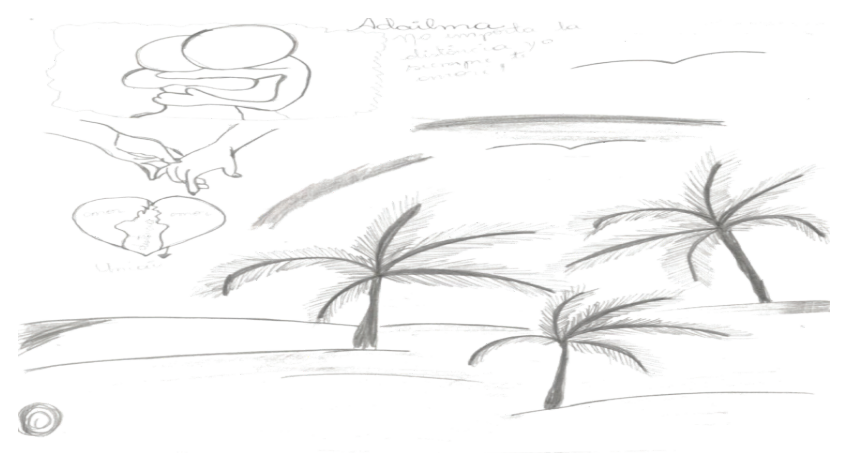

Fuente: Alumna del módulo V - Letras y Literatura Hispánica - IFRR - 2017.2

\section{CONCLUSIONES Y REFLEXIONES}

Gracias a la investigación hecha es posible concluir que el estudio del proceso de enseñanza y aprendizaje de manera general y específica de la lengua española ayuda a comprender mejor los factores que intervienen en este proceso a la vez vez que estos conocimientos impulsan a realizar investigaciones en el aula, observar y analizar las dificultades y los progresos de los alumnos, así como también a autocorregirse cuando se toma conciencia de los problemas que se afrontan como aprendientes de lenguas extranjeras ( español/inglés).

Este estudio ha hecho reflexionar a quienes escriben sobre las estrategias didácticas que se maneja cuando se está involucrado en el proceso de enseñanza y aprendizaje de una lengua extranjera, en este caso la lengua española, teniendo siempre presente la idea de competencia comunicativa que es "un conocimiento intuitivo, no consciente de una extraordinaria complejidad y abstracción, que nos permite comunicarnos con eficacia en una lengua determinada" (Baralo, 1998, p. 15).

Basadas en su experiencia docente de más de veinte años impartiendo clases las autoras pueden afirmar que la mejor manera de aprender una LE es saber qué elementos forman parte de este proceso. Por lo tanto, se vuelve muy importante que el profesor de lenguas tenga conocimiento de lo que ocurre en la mente de sus alumnos durante las clases para que puedan ser capaces de comprender, monitorizar y aceptar las estrategias utilizadas por sus alumnos cuando tienen como meta el aprendizaje en esta lengua extranjera. Asimismo es fundamental involucrarse en investigaciones que permitan brindar a los alumnos un aprendizaje significativo, eficaz y autónomo con estrategias didácticas eficaces capaces de mejorar la labor docente.

\section{REFERENCIAS BIBLIOGRÁFICAS}

Baralo, M. (1998). Teorías de adquisición de lenguas extranjeras y su aplicación a la enseñanza del español. Madrid: Fundación Antonio de Nebrija.

Baralo, M. (1999). La adquisición del español como lengua extranjera. Madrid: Arcolibro.

Baralo, M. (2000).El desarrollo de la expresión oral en el aula de E/LE. Segunda etapa CARABELA, $47,5-36$.

Baralo, M. y S. Estaire. (2010). Tendencias metodológicas postcomunicativas. En Abelló, Ehlers y Quintana (Eds.). Escenarios bilingües: el contacto de Lenguas en el Individuo y la Sociedad. Bern: Peter Lang.

Beltrán, Blanca Aguirre. (1994). Español por Profesiones. Madrid: Alcovendas.

Carabela. (2000). El desarrollo de la expresión oral en el aula E/LE. España: SGEL.

Consejo de Europa. (2002). Marco Común Europeo de Referencia para las Lenguas: aprendizaje, enseñanza, evaluación. Madrid, Secretaría General Técnica del MEC, Instituto Cervantes y Editorial Anaya. Recuperado de: http://cvc.cervantes.es/ obref/marco/

Centro Virtual Cervantes - CVC. (1997-2017). Antologías de textos de didáctica del español. El enfoque por tareas. Recuperado de: https://cvc. cervantes.es/enseñanza/biblioteca_ele.htm.

Candlin, C. (1990). Hacia la enseñanza del lenguaje mediante tareas en Comunicación, Lenguaje y Educación. (7-8. pp. 33-53). (original en inglés, 1987).

Elliot, J. (1993). El cambio educativo desde la investigación-acción. Madrd: Ediciones Morata.

Estaire, S. (1999). Tareas para hacer cosas en español: Principios y prácticas de la enseñanza de 
lenguas extranjeras mediante tareas. Colección Aula de Español. Madrid: Universidad Antonio de Nebrija.

Estaire, S. (2009). El aprendizaje mediante tareas: de la programación al aula. Madrid: Edinumen.

Estaire, S. (2009a y 2009b). El enfoque por tareas: de la fundamentación teórica a la organización de materiales didácticos y el enfoque por tareas: aspectos metodológicos y ejemplos de unidades didácticas .Antología de textos de didácticas del español. Recuperado de: http://cvccervantes.es/ enseñanza/biblioteca_ele/antología_didáctica/ default.htm.

Estaire, S. y Zanón, J. (1990). El diseño de unidades didácticas mediante tareas: principios y desarroIlo. Comunicación, Lenguaje y Educación, 7.

Fabregat, A. y Reig, D. (1998). O Sujeito como Sistema Cognitivo Processador de Informações e Construtor de Significados. (Trad. Juan Acuña Llores). En Minguet, Pilar A. (Org), A Construção do Conhecimento na Educação. Porto Alegre: ArtMed.

Gómez de Enterría, J. (2006). Comunicar y enseñar a comunicar el conocimiento especializado. Madrid: Instituto Cervantes.

Hymes, D.H. (1971). Acerca de la Competencia Comunicativa. En: Competencia Comunicativa documentos básicos en la enseñanza de lenguas extranjeras (pp. 27-46). España: Edelsa Grupo Didascalia, S.A.

Instituto de Educação, Ciência e Tecnologia de Roraima (IFRR). - Histórico da Instituição. Recuperado de: hhttp://www.ifrr.edu.br/acessoainformacao/ institucional/histórico

Instituto Brasileiro de Geografia e Estatística (IBGE). (2014). Población de Roraima /Boa Vista. Recuperado de: https://cidades.ibge.gov.br/brasil/rr/ boa-vista.

Mancera, A. M. (Ed.). (2006). Lingüística Aplicada a la Enseñanza de Español como Lengua Extranjera: Desarrollos recientes. Alcalá de Henares: Universidad de Alcalá.

McKernan, J. (1999). Investigación-Acción y curriculum: Métodos y recursos para profesionales reflexivos. Madrid: Ediciones Morata, S. L.
Miras, M. (2003). Um Ponto de Partida para a Aprendizagem de Novos Conteúdos: os conhecimentos prévios (Trad. Claudia Schilling)._O Construtivismo na Sala de Aula. (obra colectiva) São Paulo: Editora Ática.

Nunan, D. (1998). El diseño de tareas para la clase comunicativa. Madrid: CUP.

Nunan, D. (2004). Task-Based Language Teaching. Cambridge University Press.

Pozo, J. I. (1998). Teorias cognitivas de Aprendizagem. Porto Alegre: Llorens.

Secretaria de Estado do Planejamento e Desenvolvimento de Roraima (SEPLAN). (2014). Informações Socioeconômicas do Município de Boa Vista - $R R, 4^{\mathrm{a}}$ ed. (versión electrónica). Recuperado de: www.seplan.rr.gov.br.

Sansoles, F.L (2010). Enfoque de Acción: Aprender lenguas con "Tareas". En: Enfoque por Tareas. Serie Didáctica (5-30).Consejería y Embajada de España. Brasilia: Secretaria General Técnica.

Sánchez, J. G. E. (2006).últimos enfoques en la enseñanza-aprendizaje del español con fines profesionales. En Lingüística Aplicada a la Enseñanza de Español como Lengua Extranjera: Desarrollos recientes. Ana María Cestero Mancera (ed. lit.) (pp.47-60) Alcalá de Henares: Universidad de Alcalá.

Stenhouse, J. (1998). La investigación como base de la enseñanza. Madrid: Morata.

Secretaria de Planeamiento del Estado de RoraimaSEPLAN. (2014). Recuperado de: https:// informaciones economicas.gov.br/Brasil/rr/boa vista.

Wimmer, R. y Dominick, J. (2001). Introducción a la Investigación de Medios Masivos de comunicación. (6 $6^{a}$ ed.). México: Internacional Thomson Editores.

Zanón, J. (1999). La enseñanza del español mediante tareas. Madrid: Edinumen. 


\section{ANEXOS}

Anexo 1: Unidad Didáctica elaborada por las autoras

\section{Microtarea 1}

1.1 Antes de escuchar la canción, intenta completar los espacios en blanco con las palabras correspondientes a las siguientes definiciones:

1. No se emplea sino antepuesto a nombres masculinos. Es antónimo de NINGUNO.

2. Pronombre indefinido que designa persona o personas existentes, sin indicación de género ni de número. Es antónimo de NADIE.

3. Verbo transitivo que en una de sus definiciones significa "conseguir o alcanzar lo que se intenta o desea".

4. Como verbo intransitivo significa "salir victorioso".

5. Exceso de estimación propia que a veces es disimulable por nacer de causas nobles y virtuosas.

6. Artículo indeterminado en género masculino y número singular.

7. En una semana hay siete.

8. Artículo indeterminado en género femenino y número singular.

9. Pronombre indeterminado. No se emplea sino antepuesto al nombre.

10. Conjunción concesiva y adversativa que en lengua portuguesa significa: A PESAR DE / AINDA QUE.

11. El que lleva a cabo una acción heroica.

12. Verbo intransitivo que significa "alcanzar el fin o término de un desplazamiento".

\section{NO IMPORTA LA DISTANCIA}

(Ricky Martin)

\begin{tabular}{|c|}
\hline $\begin{array}{l}\text { te sentía junto a mí } \\
\text { sé que estás ahí } \\
\text { que te encontraré } \\
\text { aunque tarde una vida } \\
\text { yo jamás renunciaré } \\
\text { Un día llegaré... } \\
\text { Más allá de toda gloria } \\
\text { del orgullo y el valor } \\
\text { el poder de un (11) } \\
\text { está en su corazón } \\
\text { un día (12) } \\
\text { no importa la distancia } \\
\text { junto a ti estaré / con tu } \\
\text { resplandor } \\
\text { paso a paso iré / y per- } \\
\text { sistiré } \\
\text { a cualquier distancia } \\
\text { yo tu vida y tu amor } \\
\text { tendré. }\end{array}$ \\
\hline
\end{tabular}

\section{Microtarea 2}

2.1 Ahora escucha la canción y comprueba si lo que has escrito es correcto. Luego saca de la canción 10 palabras o expresiones que en tu opinión son importantes para la compresión del mensaje que transmite.

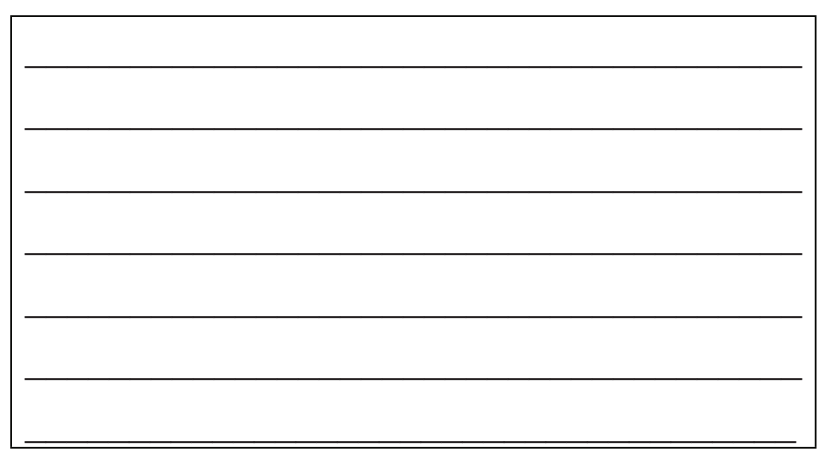




\section{Microtarea 3}

3.1 Elabora un párrafo con las palabras o expresiones que sacaste del texto.

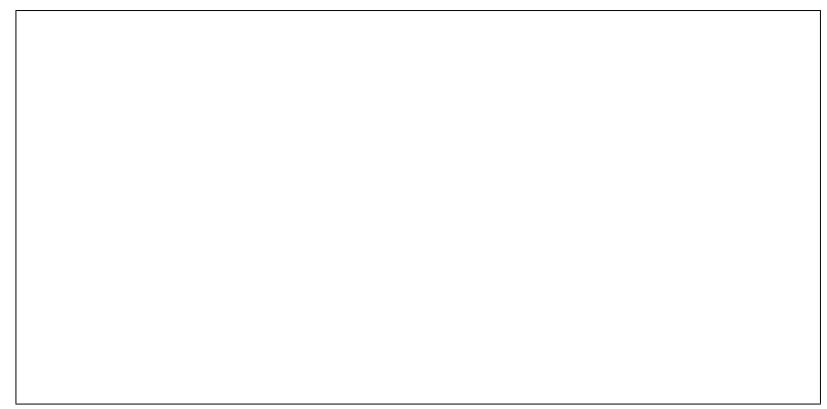

\section{Microtarea 4}

4.1 En esta canción:

1. "El rumbo encontraré" significa:

- Cualquier agujero que se hace o se produce en el casco de la nave.

- Camino y senda que uno se propone a seguir para llegar a un lugar o fin determinado.

- Pompa, ostentación y aparato costoso.

2. "Aunque tarde una vida" significa:

- Últimas horas del día.

- Tiempo que hay desde mediodía hasta anochecer.

- $\quad$ Tiempo futuro relativamente lejano.

4.2. Haz frases siguiendo el modelo comparativo: más... que)

La humildad - importante - orgullo - La humildad en más importante que el orgullo.

\begin{tabular}{|c|}
\hline El - corazón - héroe - fuerte - distancia \\
\hline La persistencia - importante - renuncia \\
\hline
\end{tabular}

Tarea Final: Producir un texto ilustrativo con dibujos, imaginando que la canción es una carta que has recibido de un (a) gran amigo (a). Presentar a los demás participantes.

"La ociosidad es la madre de todos los vicios"

$$
\text { ¡Éxito! }
$$

\section{Anexo 2 - Tarea Final presentada por los alumnos}
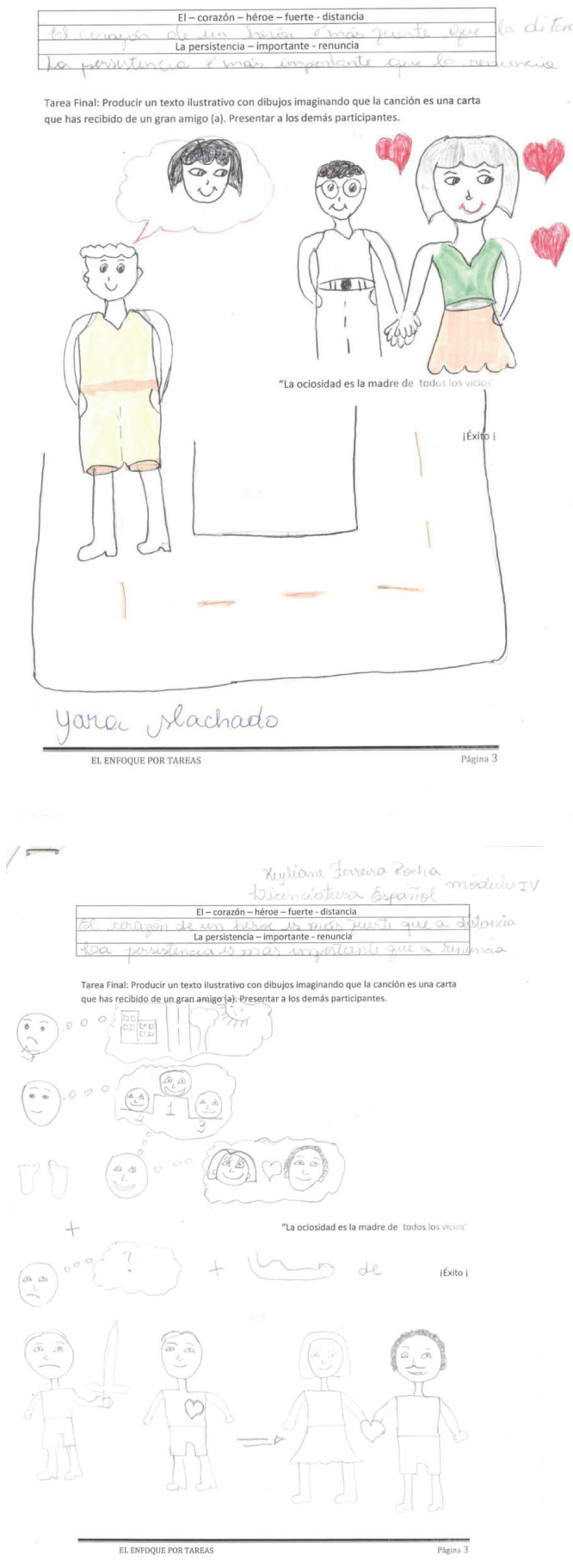
La competencia comunicativa con fines profesionales desarrollada mediante tareas

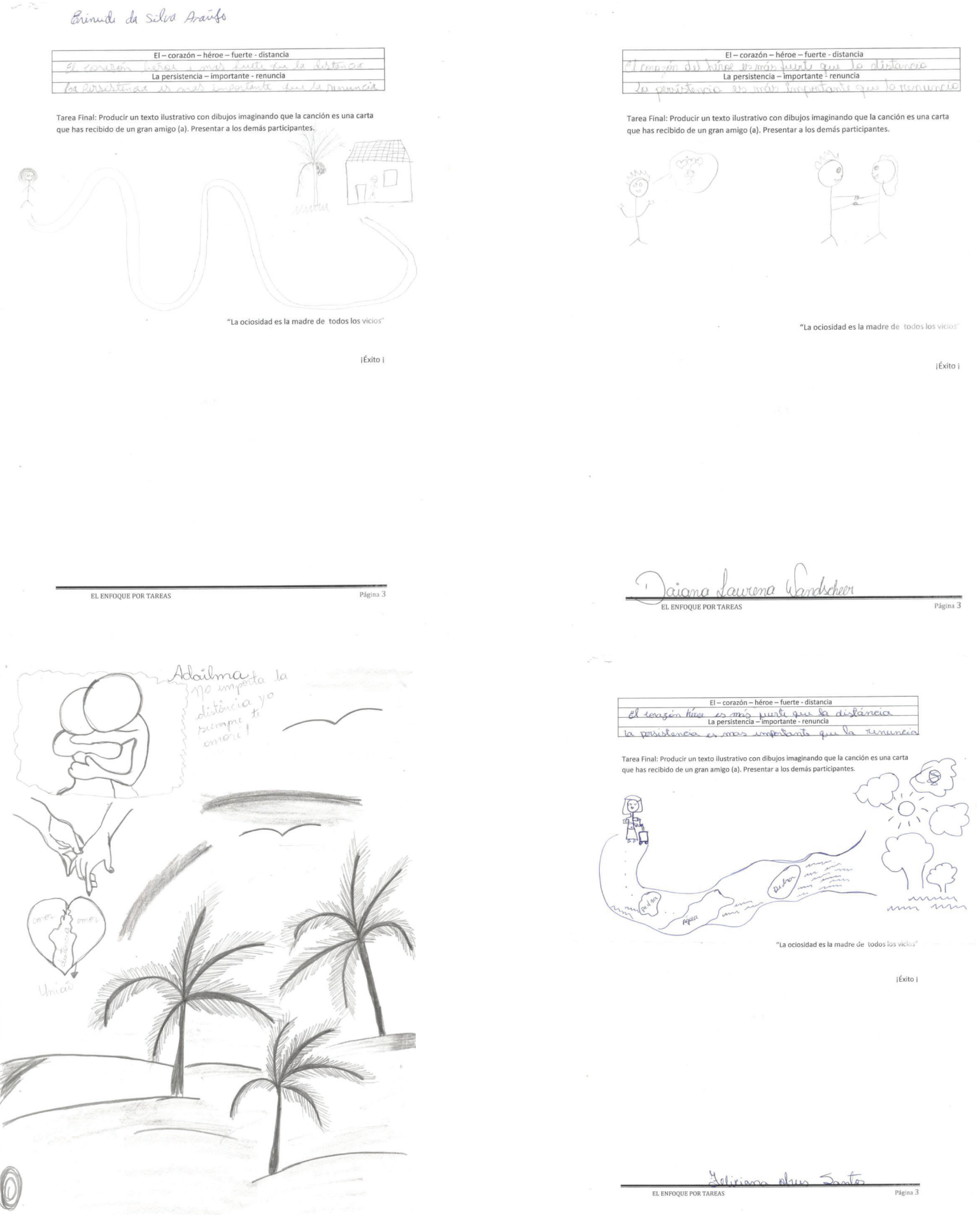


Revista Comunicación. Volumen 26, año 38, núm. 2, julio - diciembre, 2017 (pp. 63-77)

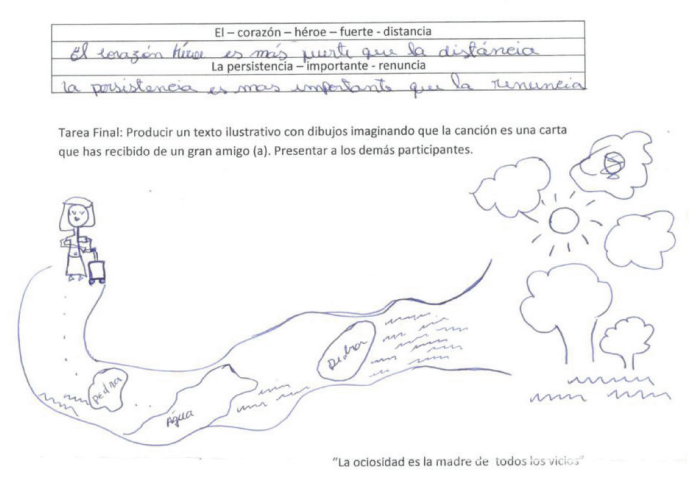

iÉxito :

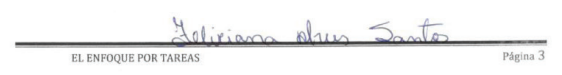

Luenuide Sousa Bontes Bunardo undulo IV - Pró vidathália

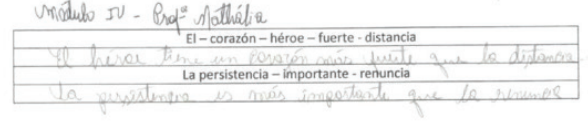

Tarea Final: Producir un texto ilustrativo con dibujos imaginando que la canción es una carta que has recibido de un gran amigo (a). Presentar a los demás participantes.

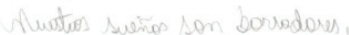

el Original istá en las manes de Dios

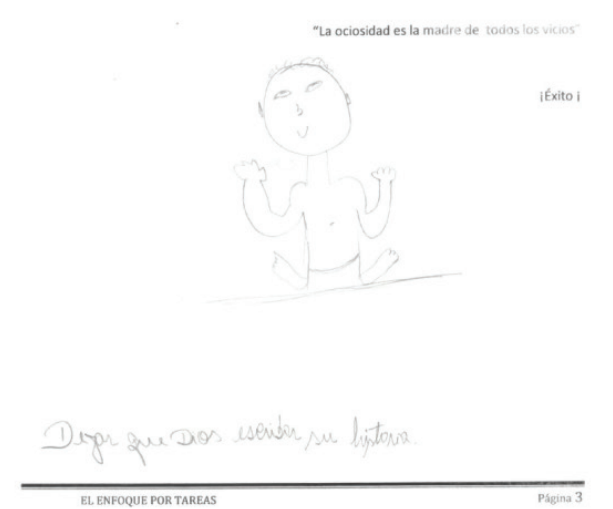

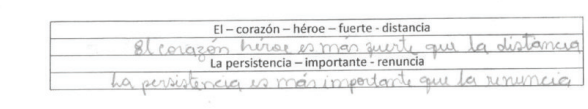

Tarea Final: Producir un texto ilustrativo con dibujos imaginando que la canción es una carta que has recibido de un gran amigo (a). Presentar a los demás participantes.

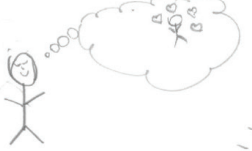

人
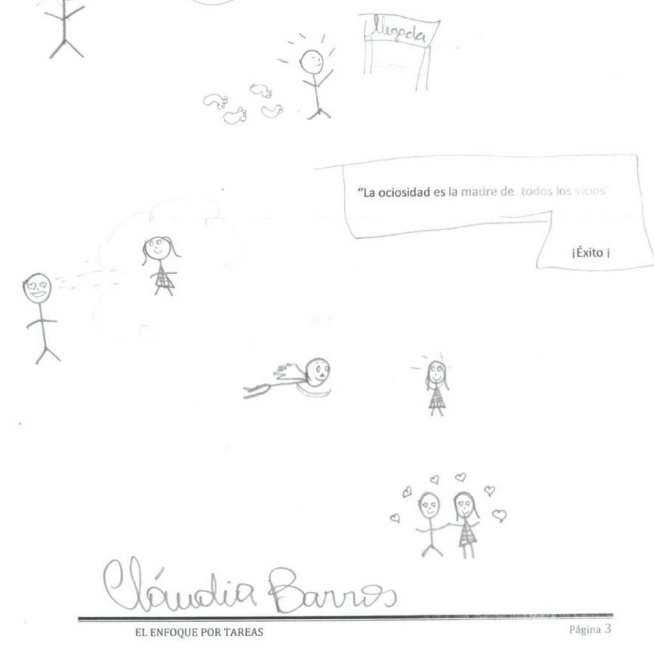

Marcien on. Reis

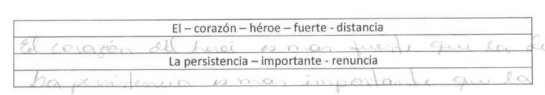

Tarea Final: Producir un texto ilustrativo con dibujos imaginando que la canción es una carta que has recibido de un gran amigo (a). Presentar a los demás participantes.

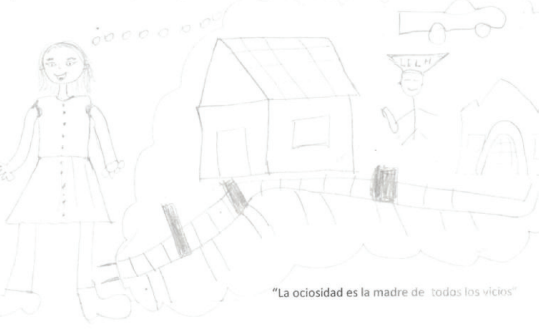

iexito i 
La competencia comunicativa con fines profesionales desarrollada mediante tareas

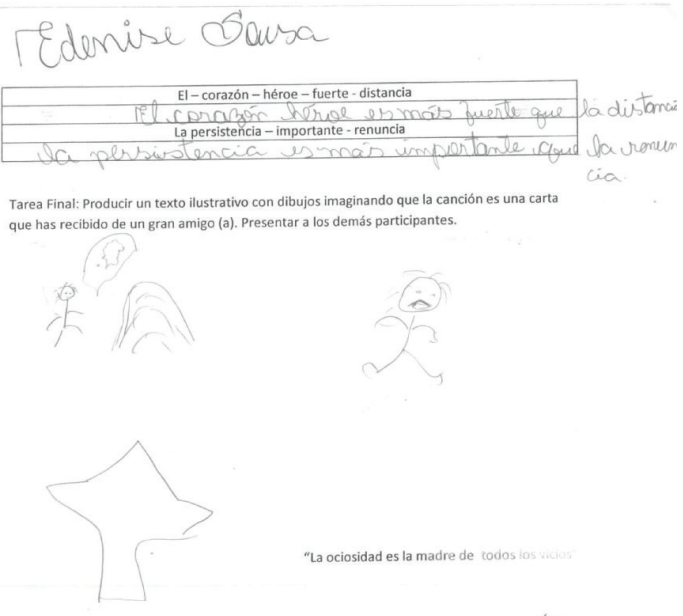

iExito :

$\overline{\text { ELENFoQUE POR TAREAS }}$

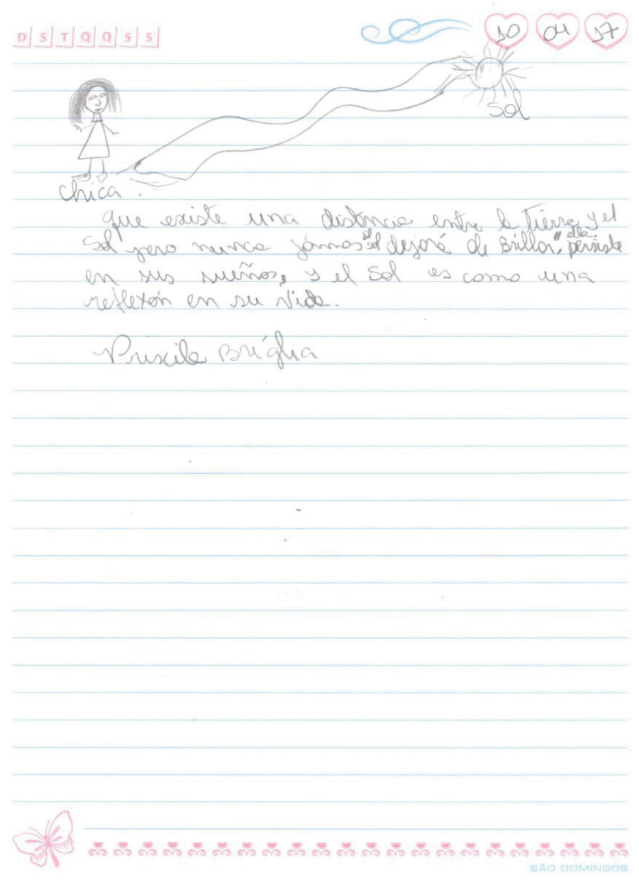

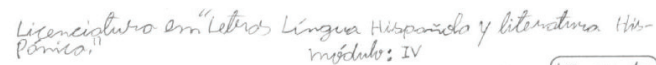
Yosé Paulo Brito de Melo módulo: Iv

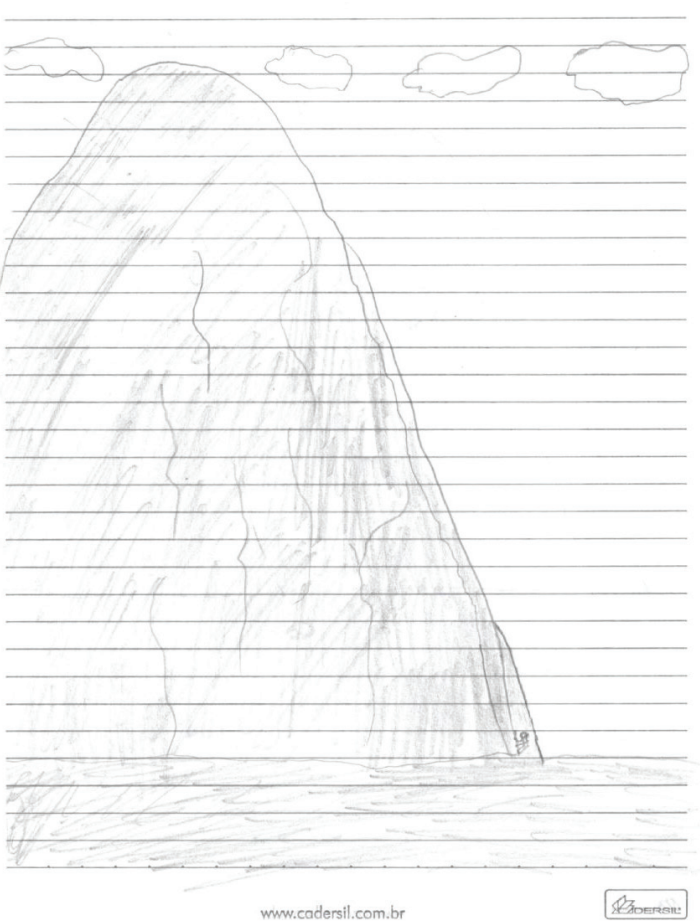


Anexo 3 - Registro de los alumnos en el aula desarrollando las tareas

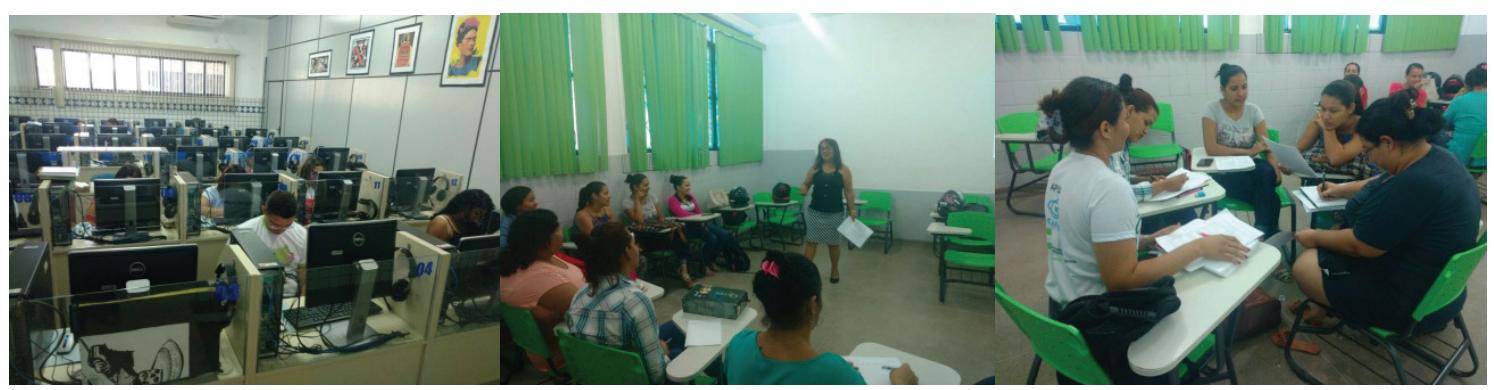

Fuente: elaboración propia 